This is a post-peer-review, pre-copyedit version of an article published in 'Mindfulness'. The final authenticated version is available online at: https://doi.org/10.1007/s12671-016-0555-3. The following terms of use apply: https://www.springer.com/gp/open

\title{
Coping Self-Efficacy and Mindfulness in Non-Suicidal Self-Injury
}

\section{Abstract}

The present study examined relationships between dispositional mindfulness, coping self-efficacy, and non-suicidal self-injury (NSSI) in 97 first-year university students $\left(78.35 \%\right.$ female; $M_{\text {age }}=18.13$ years; $\left.S D=0.81\right)$. Participants were grouped according to whether they indicated engagement in NSSI within the last 12 months, or never having engaged in NSSI, resulting in a recent NSSI group $(n=35)$, and a comparison group $(n=62)$. Participants completed the Mindful Attention Awareness Scale (MAAS), the Coping Self-Efficacy Scale's (CSES) problem-focused and emotionfocused subscales, and the Inventory of Statements About Self-Injury (ISAS). Results obtained from Pearson's correlation analysis revealed that mindfulness was significantly and positively associated with students' perceived level of coping self-efficacy. Furthermore, students who reported having engaged in NSSI in the last 12 months (i.e., those in the recent NSSI group) reported significantly lower mindfulness and lower coping self-efficacy when compared to students with no NSSI. Interestingly, coping selfefficacy was found to fully mediate the relationship between dispositional mindfulness and NSSI. The present study shows preliminary evidence for the role of coping selfefficacy in explaining the relation between mindfulness and NSSI. Implications for future research and practice regarding mindfulness as a protective factor for NSSI via coping self-efficacy are discussed.

\section{Introduction}


This is a post-peer-review, pre-copyedit version of an article published in 'Mindfulness'. The final authenticated version is available online at: https://doi.org/10.1007/s12671-016-0555-3. The following terms of use apply: https://www.springer.com/gp/open

Non-suicidal self-injury (NSSI), the self-directed deliberate destruction of body tissue without suicidal intent and for purposes not socially sanctioned, is a serious problem among young adults (Heath and Nixon 2009). Approximately $40 \%$ of individuals with self-injury from community samples have reported that their first time engaging in NSSI occurred between 17 and 24 years, suggesting that the university years are a critical period in which a considerable amount of young adults who self-injure will begin to engage in NSSI (Heath et al. 2008; Whitlock et al. 2006). Those who engage in NSSI often report using it as a coping mechanism to manage overwhelming negative thoughts or emotions (e.g., Favazza 1998; Muelhenkamp and Gutierrez 2004; Simeon and Favazza 2001). The use of NSSI as a coping strategy is specific to NSSI, whereas suicidal behaviors are typically not engaged in repeatedly over the long term to feel better or cope but rather to end one's life (Muehlenkamp 2005). Thus, the study of NSSI and perceptions of coping is of particular interest.

In university students, $12-38 \%$ report having engaged in NSSI at least once in their lifetime, and approximately $35 \%$ of university students who report a lifetime history of self-injury also report recent NSSI engagement (i.e., in the past 12 months; Favazza et al. 1989; Gratz et al. 2002). Engagement in self-injury is associated with a number of mental health problems such as anxiety, depression, eating disorders, substance abuse (Muehlenkamp 2005), suicidal ideation (Gollust et al. 2008), and emotion dysregulation (Heath et al. 2008). Given the high prevalence of self-injury in university students and the associated difficulties, it is important to determine possible protective factors related to self-injury. 
This is a post-peer-review, pre-copyedit version of an article published in 'Mindfulness'. The final authenticated version is available online at: https://doi.org/10.1007/s12671-016-0555-3. The following terms of use apply: https://www.springer.com/gp/open

Mindfulness has been suggested as a protective factor and a beneficial approach to help individuals who engage in self-injury (Garisch and Wilson 2015; Yusainy and Lawrence 2014). The structure of the construct of mindfulness has been the subject of much debate (e.g., Siegling and Petrides 2014; Tran et al. 2014). Currently, there appears to be a growing consensus that mindfulness is best understood as consisting of two dimensions: present moment awareness and acceptance or non-judgement of experience. Specifically, Kabat-Zinn (2003) defined mindfulness as being a nonjudgmental awareness and acceptance of the present moment that transpires when an individual is paying attention on purpose.

Mindfulness has been used as treatment for those who self-injure to improve emotion regulation and distress tolerance (e.g., Conterio et al. 1999; Gratz 2007; Walsh 2012). Furthermore, mindfulness is an important element of dialectical behavior therapy (DBT; a treatment for individuals with borderline personality disorder; BPD) as it focuses on the reduction of self-injurious behaviors through core mindfulness skills (e.g., nonjudgmental emotional awareness and acceptance; Gratz 2007; Linehan et al. 2006). DBT has been recognized as the most promising treatment for BPD as it is associated with positive outcomes such as fewer self-injurious, suicidal, and impulsive behaviors (Linehan et al. 2006; van den Bosch et al. 2005).

In a study examining the relationship between deficits in mindfulness and BPD features (self-harm, which subsumed both suicidal and NSSI, as well as acts of harmful dysregulated behavior) in 70 adults, Wupperman et al. (2013) found a significantly negative correlation of mindfulness with BPD features; however, in order to better 
This is a post-peer-review, pre-copyedit version of an article published in 'Mindfulness'. The final authenticated version is available online at: https://doi.org/10.1007/s12671-016-0555-3. The following terms of use apply: https://www.springer.com/gp/open

understand the mechanism by which mindfulness functions in the treatment of self-injury, an examination of the relationship between mindfulness and NSSI is required.

The ability to benefit from mindfulness interventions may be associated with a tendency to experience mindfulness in general; therefore, a number of studies have examined the role of dispositional mindfulness and its association with self-injury and other mental health outcomes (Garisch and Wilson 2015). Specifically, individuals with higher dispositional mindfulness (i.e., the experiencing of mindfulness day-to-day; Brown and Ryan 2003; Brown et al. 2007; Kabat-Zinn 2003; Murphy et al. 2012) have been shown to respond to mindfulness training with greater reported increases in mindfulness, empathy, stress reduction, and well-being compared to individuals with lower dispositional mindfulness (Shapiro et al. 2011). Furthermore, higher reports of dispositional mindfulness have also been found to be associated with greater self-reported health (Stillman et al. 2014), fewer anxious and depressive symptoms (e.g., Brown and Ryan 2003; Cash and Whittingham 2010; Rasmussen and Pidgeon 2011), and less negative affect (Arch and Craske 2010) than those reporting less dispositional mindfulness.

There is some research demonstrating that individuals' reports of dispositional mindfulness are negatively associated with self-harm in adolescents and adults (Lundh et al. 2007; Yusainy and Lawrence 2014) and NSSI in adolescents (Garisch and Wilson 2015). In a study examining the relation between deliberate self-harm (i.e., cutting, burning, severe scratching, punching oneself) and mindfulness in 123 Swedish adolescents (39.02\% female), Lundh et al. (2007) discovered that youth reporting self- 
This is a post-peer-review, pre-copyedit version of an article published in 'Mindfulness'. The final authenticated version is available online at: https://doi.org/10.1007/s12671-016-0555-3. The following terms of use apply: https://www.springer.com/gp/open

harm $(n=51)$ indicated lower levels of mindfulness compared to participants who did not engage in self-harm $(n=72)$.

Furthermore, in Yusainy and Lawrence's recent study examining the association between dispositional mindfulness, self-control, aggression, and deliberate self-harm in 241 adults (63.07 \% female), a positive relationship between mindfulness and self-control and a negative correlation between mindfulness and self-harm were found. Participants who reported engagement in self-harm also reported lower levels of mindfulness and selfcontrol, and self-control was found to mediate the association between mindfulness and self-harm.

Although both of these studies suggest that mindfulness is associated with the broad category of self-harming behaviors, it is hard to determine if there is a differential relationship between the suicidal versus non-suicidal behaviors subsumed under the broad category of self-harm. In a recent study examining the relation between mindfulness and NSSI specifically, Garisch and Wilson (2015) found that dispositional mindfulness was negatively correlated with reports of current (past 3-8 months) and lifetime history of NSSI; however, Garisch and Wilson's findings of the relationship between dispositional mindfulness and NSSI are limited to mid-adolescence (age 16 years), over a period of approximately 5 months. In light of research indicating that $40 \%$ of adolescents stop self-injuring within 1 year of starting (Whitlock et al. 2006), it is possible that many of these participants would have been engaging in a transitory short period of self-injury. Therefore, it is important to investigate NSSI in an older age group to understand factors associated with self-injury in these samples. 
This is a post-peer-review, pre-copyedit version of an article published in 'Mindfulness'. The final authenticated version is available online at: https://doi.org/10.1007/s12671-016-0555-3. The following terms of use apply: https://www.springer.com/gp/open

According to the literature, high levels of mindfulness are associated with the ability to regulate emotions (Baer et al. 2004; Baer et al. 2006; Vujanovic et al. 2010), and a number of mindfulness-based interventions are intended to enhance emotion regulation (Brown et al. 2007; Chambers et al. 2009). Emotion regulation involves the awareness, understanding, and acceptance of emotions - in addition to controlling impulsivity and using effective strategies to regulate emotions - and emotion dysregulation is considered to be the greatest risk factor for NSSI in youth and young adults (Gratz and Roemer 2004; Klonsky 2007). In a review of the literature on selfinjury, individuals with recent and/or past engagement in NSSI reported that coping with intense negative emotions is the primary reason for engaging in NSSI, and that prior to self-injuring, negative emotions are present, and following engagement in NSSI, relief is experienced as negative emotions dissipate (Klonsky 2007).

It is possible that dispositional mindfulness influences the presence or absence of self-injury by enhancing the ability to cope emotionally, which could be assessed through coping self-efficacy. Coping refers to one's cognitive and behavioral efforts to manage stressful situations (Lazarus and Folkman 1984), while self-efficacy is an individual's perceived confidence in his or her ability to carry out a specific task (Gist and Mitchell 1992). Coping self-efficacy (CSE) can be defined as one's confidence in reducing or eliminating psychological distress in the face of adversity and/or threatening events (Chesney et al. 2006). Greater mindfulness is associated with an improved ability to be aware and attend to emotions and tolerate distress, which in turn would facilitate the ability to select appropriate coping strategies for the situation. As such, it could be 
This is a post-peer-review, pre-copyedit version of an article published in 'Mindfulness'. The final authenticated version is available online at: https://doi.org/10.1007/s12671-016-0555-3. The following terms of use apply: https://www.springer.com/gp/open

hypothesized that the effect of mindfulness on NSSI would be mediated by the belief in one's ability to cope in the face of challenges.

In a related recent study, Luberto et al. (2014) examined whether CSE served as a mediator for mindfulness and emotion regulation in 180 university students (71\% female). As noted by Luberto et al. (2014), previous studies have found mindfulness to be associated with a variety of types of self-efficacy, such as the belief in one's ability to resist substance abuse relapse and managing pain (e.g., Britton et al. 2010; Chang et al. 2004; Cusens et al. 2010; Morone et al. 2009), but their study was the first to examine the relationship of mindfulness to CSE. The authors found that CSE partially mediated the relationship between mindfulness and emotion regulation, suggesting that the benefits of mindfulness for emotion regulation function to some significant degree through maximizing an individual's CSE. These findings are of particular interest due to the existing association between emotion regulation and NSSI.

In summary, the existing research has demonstrated that engagement in selfinjury is prevalent among university students (Favazza et al. 1989; Gratz et al. 2002). Mindfulness is considered to be a protective factor for self-injury and has been found to be associated with lower reports self-harm in both adolescents and adults (e.g., Lundh et al. 2007; Yusainy and Lawrence 2014), NSSI in adolescents (e.g., Garisch and Wilson 2015) and with acts of self-injury in individuals with BPD (Wupperman et al. 2013). Furthermore, mindfulness has been found to be associated with many different types of self-efficacy (Britton et al. 2010; Chang et al. 2004; Cusens et al. 2010; Morone et al. 2009), and very recently, CSE has been found to partially mediate the relationship between mindfulness and emotion regulation (Luberto et al. 2014), which is considered to 
This is a post-peer-review, pre-copyedit version of an article published in 'Mindfulness'. The final authenticated version is available online at: https://doi.org/10.1007/s12671-016-0555-3. The following terms of use apply: https://www.springer.com/gp/open

be a core factor in the engagement in NSSI (Gratz 2007; Gratz and Roemer 2004).

Despite the findings that mindfulness is negatively associated with self-harm in adolescents and adults, and NSSI in adolescents, the mechanism or pathway by which mindfulness is associated with the presence/absence of self-injury remains a nascent area of investigation.

In light of the relationship of mindfulness to self-efficacy in general, and Luberto et al.'s (2014) finding of the mediating role of CSE between mindfulness and emotion regulation, there remains the central question of how does mindfulness positively impact self-injury? It is our belief that CSE may be a particularly salient mediator between mindfulness and NSSI as the behavior itself is identified by those who engage in it as a coping technique; therefore, it may be argued that once an individual is able to be more mindful, tolerating distress, their belief in their ability to cope will be improved, which allows them to select healthier coping alternatives. Specifically, NSSI - in contrast to other self-harming behaviors (e.g., suicidal behaviors such as overdosing) — is more related to an individual's choice of and ability to cope; thus, examining the role of CSE may be particularly pertinent.

The present study sought to investigate the relationship between mindfulness, CSE, and NSSI in first-year university students. Specific objectives were to (a) compare the association between mindfulness and CSE in first-year university students, (b) examine the differences in students with and without NSSI reports of mindfulness and CSE, and (c) examine the indirect effect of mindfulness on NSSI through CSE. First, it was hypothesized that mindfulness would be strongly and positively associated with CSE. Second, it was hypothesized that students reporting recent engagement in NSSI will 
This is a post-peer-review, pre-copyedit version of an article published in 'Mindfulness'. The final authenticated version is available online at: https://doi.org/10.1007/s12671-016-0555-3. The following terms of use apply: https://www.springer.com/gp/open

also report significantly lower mindfulness and lower CSE compared to students without a history of NSSI. Finally, it was hypothesized that CSE would mediate the relationship between mindfulness and NSSI.

\section{Method}

\section{Participants}

Participants were drawn from a larger sample of first-year university students who initially took part in a study on stress and coping in Fall 2014 and who expressed interest in participating in a follow-up study on adjustment to university in Winter 2015 $(N=142)$. Of this larger sample, $25 \%$ of first-year university students reported having engaged in NSSI within the last 12 months and were included in the present study $(n=35)$. An additional subset of first-year university students without a history of NSSI and who also participated in the follow-up study on adjustment to university were assigned to a comparison group $(n=62)$. Groups were matched on gender and ethnicity. In addition, the decision was made to match on faculty as we felt that it was probable that students from the Faculty of Arts would differ from the students in Science, or Engineering, on the variables of interest. Participants were predominantly female (78 \%) and ranged in age from 17 to $21(M=18.13$ years, $S D=0.81)$. Most reported a program of study in the Faculty of Science (48\%), followed by the Faculty of Arts (42\%; including $13 \%$ of students in psychology), the Faculty of Engineering (7\%), and the Faculty of Music (3\%). Most students self-identified as Caucasian (70\%), with others reporting Asian (19\%), Indian (4\%), Middle Eastern (2\%), and other (5\%) ethnicities. Five participants did not report their ethnicity. 
This is a post-peer-review, pre-copyedit version of an article published in 'Mindfulness'. The final authenticated version is available online at: https://doi.org/10.1007/s12671-016-0555-3. The following terms of use apply: https://www.springer.com/gp/open

\section{Measures}

\section{Mindfulness}

The Mindful Attention Awareness Scale (MAAS; Brown and Ryan 2003) is a self-report unidimensional measure of trait mindfulness that has been validated in university students and community samples of adults. In a review of instruments used to measure mindfulness, it was noted that the MAAS is the most widely used mindfulness measure with consistent quality ratings for its psychometric properties (Park et al. 2013). Specifically, this measure consists of 15 items that focus on the lack of mindful attention and awareness and takes less than 5 min to complete. Items include statements such as "I find it difficult to stay focused on what's happening in the present" and "I rush through activities without being really attentive to them," and participants rate their responses on a 6-point Likert scale ranging from $1=$ almost always to $6=$ almost never. Mindfulness is indicated by lower scores or absence of inattention. Brown and Ryan (2003) make the argument that for those who are not mindful, it is easier to respond about inattention or lack of present-moment awareness than questions directly assessing mindfulness in the moment. The MAAS has high internal consistency with Cronbach alphas ranging from 0.80 to 0.90 , high test-retest reliability, as well as discriminant, convergent, and criterion validity (Brown and Ryan 2003). In the current sample, the MAAS had high internal consistency $(\alpha=0.87)$. The selection of a unidimensional measure of mindfulness was made for two reasons; first, in a review of existing measures, the MAAS had the strongest psychometric properties for any measure of comparable length; second, the 
This is a post-peer-review, pre-copyedit version of an article published in 'Mindfulness'. The final authenticated version is available online at: https://doi.org/10.1007/s12671-016-0555-3. The following terms of use apply: https://www.springer.com/gp/open

focus on inattention was found in piloting to be easier to comprehend by university students with no experience with mindful practice.

\section{Coping Self-Efficacy}

The Coping Self-Efficacy Scale (CSES; Chesney et al. 2006) is a self-report measure designed to assess perceived self-efficacy for coping with challenging situations (Bandura 1997). The CSES contains 26 items and three subscales: (a) problem-focused coping (6 items), (b) emotion-focused coping (4 items), and (c) social support (3 items). For the present study, which focused on mindfulness and NSSI, it was decided that only items pertaining to problem-focused coping (e.g., "Find solutions to your most difficult problems," "Sort out what can be changed, and what cannot be changed") and emotionfocused coping (e.g., "Stop yourself from being upset by unpleasant thoughts," "Keep from feeling sad") were most critical to this relationship; thus, only these two subscales were included. Participants rate how confident they are that they can employ these coping behaviors on an 11-point Likert scale ranging from $0=$ cannot do at all to $10=$ certain can do. Higher scores on the CSES represent greater CSE. The CSES is negatively correlated with perceived stress, burnout (Chesney et al. 2006), cognitive state and somatic state anxiety (Nicholls et al. 2010), and emotion regulation difficulties (Luberto et al. 2014). In contrast, CSES is positively correlated with optimism (Chesney et al. 2006). In the present study, the CSES was found to be highly reliable (21 items; $\alpha=0.93$ ).

\section{Non-Suicidal Self-Injury}


This is a post-peer-review, pre-copyedit version of an article published in 'Mindfulness'. The final authenticated version is available online at: https://doi.org/10.1007/s12671-016-0555-3. The following terms of use apply: https://www.springer.com/gp/open

The Inventory of Statements About Self-Injury (ISAS; Klonsky and Glenn 2009) is a self-report measure that assesses the frequency (section 1) and functions (section 2) of NSSI behaviors in youth and young adults (e.g., Glenn and Klonsky 2011; Hamza and Willoughby 2014). In the first section of the ISAS, participants indicate lifetime frequency and duration of 12 NSSI behaviors (e.g., cutting, banging/hitting, biting, burning, and carving) and estimate number of times of intentional self-injurious behavior across their lifetime (e.g., 0, 10, 100, 500 times). Participants who report at least one NSSI behavior are prompted to complete questions regarding descriptive features and contextual factors of NSSI (e.g., age of onset, date of most recent episode, experience of pain during NSSI, privacy of the behavior).

\section{Procedure}

Participants were recruited from undergraduate classes at a large Canadian university. Once permission was granted to visit undergraduate classes, research assistants (RAs) introduced the project and its purposes at the beginning of class and distributed an envelope to all students present in class. Students were told (orally and on the consent form) that their participation was completely voluntary and would have no effect on their grades. Students were provided with an envelope that contained (a) a consent form, (b) a screening questionnaire, (c) a contact information sheet, and (d) a debriefing information sheet with resources for further support. A unique participant number identified each envelope and documents. Envelopes were collected at the end of each class. Students who provided their contact information in Fall 2014 were invited via e-mail to participate in a follow-up study on adjustment to university during Winter 2015. 
This is a post-peer-review, pre-copyedit version of an article published in 'Mindfulness'. The final authenticated version is available online at: https://doi.org/10.1007/s12671-016-0555-3. The following terms of use apply: https://www.springer.com/gp/open

Students who expressed interest in participating in this follow-up study were sent an individualized link and password to access an online survey that included a series of confidential questionnaires, including the MAAS, CSES, and ISAS. They were able to view a consent form with information about the study, confidentiality, and ability to withdraw at any time without penalty, prior to completing any of the questionnaires. Once the questionnaires were completed, participants received an e-mail thanking them for their participation in the study, in addition to debriefing information, additional resources, and research compensation. The initial study and follow-up study were approved by the university's Institutional Ethics Review Board.

\section{Data Analytic Plan}

\section{Descriptive Statistics and Correlations}

Prior to conducting analyses, all continuous variables were examined through SPSS for accuracy of data entry, missing values, and fit between their distributions and assumptions of multivariate analyses. All variables were normally distributed, with skewness and kurtosis values below 1.0. Pearson's correlations were also conducted to examine the strength and direction of the associations between variables of interest. Given the relatively strong association between mindfulness and CSE, a multivariate analysis of variance was used to assess group differences.

\section{Group Differences}

One multivariate analysis of variance (MANOVA) was conducted to measure group differences on mindfulness and CSE. To address variation in group sizes, Pillai's trace rather than Wilks' lambda was used for comparisons (Tabachnick and Fidell 2013). 
This is a post-peer-review, pre-copyedit version of an article published in 'Mindfulness'. The final authenticated version is available online at: https://doi.org/10.1007/s12671-016-0555-3. The following terms of use apply: https://www.springer.com/gp/open

\section{Simple Mediation Analysis}

The simple mediation analysis was tested with the bootstrapping technique (Hayes 2009; Preacher and Hayes 2004). This method employs data-resampling procedures to estimate the direct and indirect effects of a given mediator and is recommended in studies using a small sample size (MacKinnon et al. 2004). Given that the mediation model included a continuous predictor variable (i.e., mindfulness), a continuous mediator variable (i.e., CSE), and a dichotomous outcome variable (i.e., NSSI status), a logistic regression-based path analytic framework using the SPSS computer tool "PROCESS" (Hayes 2012) was used.

A linear regression analysis was first conducted to establish the role of mindfulness on CSE (path a). Then, one logistic regression was conducted to examine the role of CSE on NSSI status (path b). Finally, one additional logistic regression was conducted to examine the direct effect of mindfulness on NSSI status (path c) and the indirect effect of mindfulness on NSSI status through CSE by adding this indirect effect path into the equation (path c'). Bias-corrected bootstrapped confidence intervals were used to confirm the statistical significance of the indirect effect of CSE on the relationship between mindfulness and NSSI status. The indirect effect is significant only if the upper and lower confidence interval does not include zero (Preacher and Hayes 2004).

\section{Results}

In the current study, 35 students reported having engaged in NSSI in the last 12 months. The majority of students $(n=23 ; 66 \%)$ started to self-injure between the ages of 
This is a post-peer-review, pre-copyedit version of an article published in 'Mindfulness'. The final authenticated version is available online at: https://doi.org/10.1007/s12671-016-0555-3. The following terms of use apply: https://www.springer.com/gp/open

12 and 15 years old $\left(M_{\mathrm{age}}=14.54, S D=2.08\right)$; yet, $20 \%(n=7)$ had a later onset and started between 17 and 18 years old. Although most participants endorsed more than one NSSI behavior in the present study, $41 \%$ identified cutting as their main form of selfinjury, followed by banging or hitting oneself (18\%), severe scratching (15\%), rubbing skin against rough surface $(12 \%)$, and other (3\%). Five students did not specify their main form of NSSI.

CSE was significantly and positively associated with mindfulness $(r=0.52$, $p<0.01)$. Table 1 displays means and standard deviations between variables in the total sample, NSSI group, and comparison group.

A one-way MANOVA was conducted to determine if students' perceived level of mindfulness and CSE differed based on their NSSI status. Preliminary assumption checking revealed that data was normally distributed and did not include any univariate or multivariate outliers. Furthermore, there were linear relationships between variables of interest and equal variance-covariance matrices, as assessed by Box's M test $(p=0.30)$. There was a statistically significant difference in perceived mindfulness and CSE based on students' NSSI status, $F(2,72)=6.10, p<0.01, \eta^{2}=0.15$. More specially, university students who engaged in NSSI in the last 12 months reported significantly lower levels of mindfulness $\left(F(1,73)=6.81, p<0.05, \eta^{2}=0.09\right)$ and $\operatorname{CSE}(F(1,73)=10.86, p<0.01$, $\eta^{2}=0.13$ ) than those with no history of NSSI. The indirect effect of mindfulness on NSSI status through CSE was examined with the bootstrapping technique (Hayes 2009; Preacher and Hayes 2004). As displayed in Fig. 1a, mindfulness was regressed on NSSI status with a logistic regression. The logistic regression model was statistically significant, $\chi^{2}(1)=5.90, p<0.05$, and revealed that mindfulness explained $11 \%$ 
This is a post-peer-review, pre-copyedit version of an article published in 'Mindfulness'. The final authenticated version is available online at: https://doi.org/10.1007/s12671-016-0555-3. The following terms of use apply: https://www.springer.com/gp/open

(Nagelkerke R 2) of the variance in NSSI status. Then, as depicted in Fig. 1b, mindfulness was regressed on perceived self-efficacy through a linear regression, $\beta=1.53, t=5.08, p<0.001$, and accounted for $27 \%$ of its variance. Finally, a second logistic regression was performed to examine the indirect effect of mindfulness on NSSI status through CSE. The logistic regression model was statistically significant, $\chi^{2}(2)=10.45, p<0.05$ and explained $19 \%$ (Nagelkerke R 2) of the variance in NSSI status. While CSE was a significant predictor of NSSI status, mindfulness was no longer one after CSE was entered in the model, therefore indicating a full mediation. A biascorrected bootstrap confidence interval was generated based on 1000 bootstrap samples to test the statistical significance of the mediating effect of CSE on the relationship between mindfulness and NSSI status. Given the confidence interval $(95 \% \mathrm{CI}[-0.0736$ to -0.0028$]$ ) was entirely below zero, there is evidence that CSE fully mediated the mindfulness-NSSI status relationship. Specifically, low mindfulness was related to poor CSE, which in turn was related to a greater likelihood of NSSI engagement. Table 2 includes the regression weights, significance tests, and confidence intervals for the variables in the mediation model.

\section{Discussion}

Previous research has documented the association between mindfulness and CSE (Luberto et al. 2014), mindfulness and self-injury (Garisch and Wilson 2015), emotion regulation and self-injury (Klonsky 2007) and, very recently, the importance of CSE as a mediator between mindfulness and emotion regulation (Luberto et al. 2014). However, the present study was the first to examine the relationship between mindfulness, CSE (problem- and emotion-focused) and NSSI in university students. 
This is a post-peer-review, pre-copyedit version of an article published in 'Mindfulness'. The final authenticated version is available online at: https://doi.org/10.1007/s12671-016-0555-3. The following terms of use apply: https://www.springer.com/gp/open

Although cultivating mindfulness is frequently considered to be an important element of treatment for self-injury (e.g., Gratz 2007; Linehan et al. 2006), the current study is the only direct examination of the way in which mindfulness is associated with the presence/absence of self-injury. In particular, we sought to (a) explore the relationship between mindfulness and CSE in this sample of university students, (b) examine possible differences in reports of mindfulness and CSE in those with recent NSSI and their nonself-injuring peers; and building on Luberto et al.'s previous research (2014), (c) investigate the possible indirect effect of mindfulness on NSSI through the potential mediating effect of CSE.

Consistent with our hypotheses and previous literature, CSE was found to significantly and positively correlate with mindfulness. Second, as expected, those with recent NSSI reported significantly lower mindfulness and CSE than their non-selfinjuring peers. Furthermore, CSE was found to fully mediate the relationship between mindfulness and NSSI.

Our results support Luberto et al.'s (2014) finding on the relationship between self-reports of experienced mindfulness and CSE in young adults. The day-to-day experiencing of mindfulness that was measured in the present study was shown to be associated with CSE, specifically the ability to solve problems and cope with difficult emotions. Furthermore, individuals with recent NSSI reported significantly lower mindfulness and problem- and emotion-focused CSE. Although previous researchers have found lower reports of mindfulness in young adults who report self-harm as a broader construct (including suicidal and NSSI; e.g., Yusainy and Lawrence 2014), the inclusion of suicidal self-injury makes the generalization of these findings to the present 
This is a post-peer-review, pre-copyedit version of an article published in 'Mindfulness'. The final authenticated version is available online at: https://doi.org/10.1007/s12671-016-0555-3. The following terms of use apply: https://www.springer.com/gp/open

sample questionable. As noted earlier, there has only been one study that recently examined mindfulness and NSSI in a community sample of adolescents, with results demonstrating that those with NSSI reported lower mindfulness relative to their non-selfinjuring peers (Garisch and Wilson 2015). The present results provide additional support for the finding that those with NSSI report lower mindfulness; however, this is the first study to find this relationship in a university sample. Nevertheless, the lower reports of mindfulness in those with recent NSSI must be interpreted with reference to the mediation model discussed below.

The finding that the NSSI group reported significantly lower CSE, specifically problem- and emotion-focused CSE, in comparison to the non-NSSI group, is the first evidence that those who report recent NSSI indicate less confidence in their ability to use problem- and emotion-focused coping. It was hypothesized that this would be the case in light of previous research documenting that individuals who report engaging in NSSI also report greater difficulties with problem solving (e.g., Nock and Mendes 2008) as well as significant difficulties in their ability to manage intense negative emotions (e.g., Gratz and Roemer 2004; Klonsky 2007). However, the assessment of CSE directly taps into the individual's confidence in their ability to employ problem solving and emotion coping, or to control negative thoughts and emotions (e.g., to make unpleasant thoughts go away; stop yourself from being upset by unpleasant thoughts). Although the current results indicate a co-occurrence of NSSI and self-reports of poor coping ability and causality cannot be inferred, this association suggests that having poor CSE may be characteristic of NSSI. Yet, similar to the group differences in mindfulness, these differences in CSE should be considered with reference to the mediation model below. 
This is a post-peer-review, pre-copyedit version of an article published in 'Mindfulness'. The final authenticated version is available online at: https://doi.org/10.1007/s12671-016-0555-3. The following terms of use apply: https://www.springer.com/gp/open

The finding that CSE fully mediates the relationship between mindfulness and NSSI may reflect a pattern whereby the benefits of mindfulness for NSSI occur as a result of increased mindfulness leading to a greater sense of CSE, which in turn decreases risk for self-injury, although this requires replication in a longitudinal study to confirm actual causal pathways. Nevertheless, interestingly, the present role of CSE as fully mediating the relationship between mindfulness and NSSI indicates an even greater importance of CSE in the potential protective role of mindfulness relative to Luberto et al.'s (2014) study, which found that CSE partially mediated the relationship between mindfulness and emotion regulation. Although both Luberto et al. and our results suggest a pattern of mindfulness enhancing CSE and in turn positively influencing emotion regulation or NSSI, there are differences in the degree of the relationship.

One possible explanation of this difference is the more severe nature of our outcome variable, thus resulting in a stronger relationship with CSE. A second explanation could be that in the current study, the CSE variable consisted exclusively of problem- and emotion-focused CSE, which have the greatest relevance to mindfulness and self-injury, whereas Luberto et al. included the social support items that may be less related to emotion regulation and mindfulness. Despite these differences, the present findings together with Luberto et al. may be interpreted to suggest that the mechanism by which mindfulness training benefits those who self-injure is through the enhancement of their CSE, which in turn leads to better emotion regulation and thus less risk for selfinjury. As noted earlier, in community samples of individuals who report NSSI, the use of self-injury to regulate intense negative emotions is the most commonly endorsed reason for the self-injury (Klonsky 2007). Thus, if the ability to be mindful increases the 
This is a post-peer-review, pre-copyedit version of an article published in 'Mindfulness'. The final authenticated version is available online at: https://doi.org/10.1007/s12671-016-0555-3. The following terms of use apply: https://www.springer.com/gp/open

young adult's feeling of control in dealing with intense negative emotions (emotionfocused coping), then the individual would be less at risk for NSSI. As noted earlier, longitudinal studies would be needed to determine the exact causal pathway, as both the current study and Luberto et al.'s (2014) research have been limited to cross-sectional designs. Mediation analysis suggests a temporal relationship, based on prior literature, but cannot definitively determine cause. Nevertheless, the pattern of associations between mindfulness, CSE, and NSSI provides important preliminary information.

The present study has a number of limitations. First as noted above, the crosssectional design precludes causal determinations regarding the role of mindfulness and/or CSE in the occurrence of self-injury. Furthermore, it is possible that both changes in mindfulness, CSE, and associated changes in self-injury are all a result of change in a third related variable such as depression. Thus, future studies, evaluating the improved mindfulness and possible associated changes in CSE and subsequent decreases in selfinjury are needed, as well as studies including common associated variables such as depression. Second, the small number of males in the NSSI group made gender analyses impossible and the predominately Caucasian sample negated evaluation of differences by ethnicity. Third, the present study would have been strengthened by the inclusion of both a measure of CSE and of emotion regulation to better build on Luberto et al.'s (2014) work. Similarly, in the present study, the social support subscale was not included as the focus of the study was on "within-person" coping; as such, it would be interesting to examine all subscales of the CSES in future mindfulness research.

Fourth, although the MAAS is a commonly used measure of trait mindfulness that has been found to have good psychometrics in use with young adults (Brown and Ryan 
This is a post-peer-review, pre-copyedit version of an article published in 'Mindfulness'. The final authenticated version is available online at: https://doi.org/10.1007/s12671-016-0555-3. The following terms of use apply: https://www.springer.com/gp/open

2003), measures of both trait and state mindfulness would have been advantageous to more fully evaluate the role of mindfulness in NSSI. Furthermore, the MAAS is considered to be a unidimensional measure of mindfulness, primarily assessing present moment awareness; however, mindfulness increasingly has been recognized as being multidimensional including both present-moment awareness and non-judgemental acceptance of experience (e.g., Tran et al. 2014). In light of the present results, future research would benefit from a more detailed examination of the relation of the different dimensions of mindfulness to coping self-efficacy and NSSI. Finally, although mindfulness and CSE are distinct constructs, there is clearly some overlap which may have contributed to some of the present findings.

Despite the limitations in the current study, there are many significant findings with important implications for future research and clinical practice. The present results provide evidence of (a) the strong relationship between problem- and emotion-based CSE and mindfulness in a university sample, (b) lower CSE in young adults with recent NSSI, and (c) lower mindfulness in those with recent NSSI. Furthermore, the present study provides the first evidence of the complex relationship of mindfulness to CSE and NSSI in young adults. In working with young adults with self-injury, currently enhancing mindfulness is frequently an important part of treatment. Based on the present results, it would be advisable to also assess problem- and emotion-based CSE. Furthermore, in explaining the utility of mindfulness in treatment, the role of mindfulness in improving one's CSE should be discussed. Finally, the current findings contribute to our understanding of mindfulness and a possible critical pathway by which mindfulness results in the myriad of benefits in mental health-related outcomes. 
This is a post-peer-review, pre-copyedit version of an article published in 'Mindfulness'. The final authenticated version is available online at: https://doi.org/10.1007/s12671-016-0555-3. The following terms of use apply: https://www.springer.com/gp/open

\section{References}


This is a post-peer-review, pre-copyedit version of an article published in 'Mindfulness'. The final authenticated version is available online at: https://doi.org/10.1007/s12671-016-0555-3. The following terms of use apply: https://www.springer.com/gp/open

Arch, J. J., \& Craske, M. G. (2010). Laboratory stressors in clinically anxious and nonanxious individuals: The moderating role of mindfulness. Behaviour Research and Therapy. doi:10.1016/j.brat.2010.02.005.

Baer, R. A., Smith, G. T., \& Allen, K. B. (2004). Assessment of mindfulness by self-report: The Kentucky Inventory of mindfulness skills. Assessment. doi:10.1177/1073191104268029.

Baer, R. A., Smith, G. T., Hopkins, J., Krietemeyer, J., \& Toney, L. (2006). Using self-report assessment methods to explore facets of mindfulness. Assessment. doi:10.1177/1073191105283504.

Bandura, A. (1997). Self-efficacy: The exercise of control. New York: Freema.

Britton, W. B., Bootzin, R. R., Cousins, J. C., Hasler, B. P., Peck, T., \& Shapiro, S. L. (2010). The contribution of mindfulness practice to a multicomponent behavioral sleep intervention following substance abuse treatment in adolescents: a treatmentdevelopment study. Substance Abuse. doi:10.1080/08897071003641297.

Brown, K. W., \& Ryan, R. M. (2003). The benefits of being present: mindfulness and its role in psychological well-being. Journal of Personality and Social Psychology. doi:10.1037/0022-3514.84.4.822.

Brown, K. W., Ryan, R. M., \& Creswell, J. D. (2007). Mindfulness: Theoretical foundations and evidence for its salutary effects. Psychological Inquiry. doi:10.1080/10478400701598298. 
This is a post-peer-review, pre-copyedit version of an article published in 'Mindfulness'. The final authenticated version is available online at: https://doi.org/10.1007/s12671-016-0555-3. The following terms of use apply: https://www.springer.com/gp/open

Cash, M., \& Whittingham, K. (2010). What facets of mindfulness contribute to psychological well-being and depressive, anxious, and stress-related symptomatology? Mindfulness. doi:10.1007/s12671-010-0023-4.

Chambers, R., Gullone, E., \& Allen, N. B. (2009). Mindful emotion regulation: An integrative review. Clinical Psychology Review. doi:10.1016/j.cpr.2009.06.005.

Chang, V. Y., Palesh, O., Caldwell, R., Glasgow, N., Abramson, M., Luskin, F., et al. (2004). The effects of a mindfulness-based stress reduction program on stress, mindfulness selfefficacy, and positive states of mind. Stress and Health. doi:10.1002/smi.1011.

Chesney, M. A., Neilands, T. B., Chambers, D. B., Taylor, J. M., \& Folkman, S. (2006). A validity and reliability study of the coping self-efficacy scale. British Journal of Health Psychology. doi:10.1348/135910705X53155.

Conterio, K., Lader, W., \& Bloom, J. K. (1999). Bodily harm: The breakthrough healing program for self-injurers. New York: S.A.F.E. Alternatives.

Cusens, B., Duggan, G. B., Thorne, K., \& Burch, V. (2010). Evaluation of the breathworks mindfulness-based pain management programme: Effects on well-being and multiple measures of mindfulness. Clinical Psychology \& Psychotherapy. doi:10.1002/cpp.653.

Favazza, A. R. (1998). The coming of age of self-mutilation. The Journal of Nervous and Mental Disease, 186(5), 259-268.

Favazza, A. R., DeRosear, L., \& Conterio, K. (1989). Self-mutilation and eating disorders. Suicide and Life Threating Behavior. doi:10.1111/j.1943-278X.1989.tb00566.x. 
This is a post-peer-review, pre-copyedit version of an article published in 'Mindfulness'. The final authenticated version is available online at: https://doi.org/10.1007/s12671-016-0555-3. The following terms of use apply: https://www.springer.com/gp/open

Garisch, J. A., \& Wilson, M. S. (2015). Prevalence, correlates, and prospective predictors of nonsuicidal self injury among New Zealand adolescents: Cross-sectional and longitudinal survey data. Child and Adolescent Psychiatry and Mental Health. doi:10.1186/s13034015-0055-6.

Gist, M. E., \& Mitchell, T. R. (1992). Self-efficacy: A theoretical analysis of its determinants and malleability. The Academy of Management Review. doi:10.5465/AMR.1992.4279530.

Glenn, C. R., \& Klonsky, D. E. (2011). Prospective prediction of nonsuicidal self-injury: A 1year longitudinal study in young adults. Behavior Therapy. doi:10.1016/j.beth.2011.04.005.

Gollust, S. E., Eisenberg, D., \& Golberstein, E. (2008). Prevalence and correlates of self-injury among university students. Journal of American College Health. doi:10.3200/JACH.56.5.491-498.

Gratz, K. L. (2007). Targeting emotion dysregulation in the treatment of self-injury. Journal of Clinical Psychology. doi:10.1002/jclp.20417.

Gratz, K. L., \& Roemer, L. (2004). Multidimensional assessment of emotional regulation and dysregulation: Development, factor structure, and initial validation of the difficulties in emotion regulation scale. Journal of Psychopathology and Behavioral Assessment. doi:10.1023/B:JOBA.0000007455.08539.94.

Gratz, K. L., Conrad, S. D., \& Roemer, L. (2002). Risk factors for deliberate self-harm among college students. American Journal of Orthopsychiatry. doi:10.1037/002-9432.72.1.128. 
This is a post-peer-review, pre-copyedit version of an article published in 'Mindfulness'. The final authenticated version is available online at: https://doi.org/10.1007/s12671-016-0555-3. The following terms of use apply: https://www.springer.com/gp/open

Hamza, C. A., \& Willoughby, T. (2014). A longitudinal person-centered examination of nonsuicidal self-injury among university students. Journal of Youth and Adolescence. doi:10.1007/s10964-013-9991-8.

Hayes, A. F. (2009). Beyond Baron and Kenny: Statistical mediation analysis in the new millennium. Communication Monographs. doi:10.1080/03637750903310360.

Hayes, A. F. (2012). PROCESS: A versatile computational tool for observed variable mediation, moderation, and conditional process modeling [White paper]. Retrieved from http://www.afhayes.com/public/process2012.pdf. Accessed 14 Sept 2015.

Heath, N. L., \& Nixon, M. K. (2009). Assessment of nonsuicidal self-injury in youth. In SelfInjury in Youth: The Essential Guide to Assessment and Intervention (pp. 143-170). New York: Routledge.

Heath, N. L., Toste, J. R., Nedecheva, T., \& Charlebois, A. (2008). An examination of nonsuicidal self-injury among college students. Journal of Mental Health Counselling. doi:10.17744/mehc.30.2.8p879p3443514678.

Kabat-Zinn, J. (2003). Mindfulness-based interventions in context: Past, present, and future. Clinical Psychology: Science and Practice. doi:10.1093/clipsy/bpg016.

Klonsky, E. D. (2007). The functions of deliberate self-injury: A review of the empirical evidence. Clinical Psychology Review. doi:10.1016/j.cpr.2006.08.002.

Klonsky, E. D., \& Glenn, C. R. (2009). Assessing the functions of non-suicidal self-injury: Psychometric properties of the Inventory of Statements About Self-injury (ISAS). Journal of Psychopathology and Behavioral Assessment. doi:10.1007/s10862-008-9107-z. 
This is a post-peer-review, pre-copyedit version of an article published in 'Mindfulness'. The final authenticated version is available online at: https://doi.org/10.1007/s12671-016-0555-3. The following terms of use apply: https://www.springer.com/gp/open

Lazarus, R. S., \& Folkman, S. (1984). Stress, Appraisal, and Coping. New York: Springer.

Linehan, M. M., Comtois, K. A., Murray, A. M., Brown, M. Z., Gallop, R. J., Heard, H. L., et al. (2006). Two-year randomized controlled trial and follow-up of dialectical behavior therapy vs. therapy by experts for suicidal behaviors and borderline personality disorder. Archives of General Psychiatry. doi:10.1001/archpsyc.63.7.757.

Luberto, C. M., Cotton, S., McLeish, A. C., Mingione, C. J., \& O’Bryan, E. M. (2014). Mindfulness skills and emotion regulation: The mediating role of coping self-efficacy. Mindfulness. doi:10.1007/s12671-012-0190-6.

Lundh, L.-G., Karim, J., \& Quilisch, E. (2007). Deliberate self-harm in 15-year-old adolescents: A pilot study with a modified version of the deliberate self-harm inventory. Journal of Psychology. doi:10.1111/j.1467-9450.2006.00567.x.

MacKinnon, D. P., Lockwood, C. M., \& Williams, J. (2004). Confidence limits for the indirect effect: Distribution of the product and resampling methods. Multivariate Behavioral Research. doi:10.1207/s15327906mbr3901_4.

Morone, N. E., Rollman, B. L., Moore, C. G., Qin, L., \& Weiner, D. K. (2009). A mind-body program for older adults with chronic low back pain: results of a pilot study. Pain Medicine. doi:10.1111/j.1526-4637.2009.00746.x.

Muehlenkamp, J. (2005). Self-injurious behavior as a separate clinical syndrome. American Journal of Orthopsychiatry. doi:10.1037/0002-9432.75.2.324. 
This is a post-peer-review, pre-copyedit version of an article published in 'Mindfulness'. The final authenticated version is available online at: https://doi.org/10.1007/s12671-016-0555-3. The following terms of use apply: https://www.springer.com/gp/open

Muelhenkamp, J., \& Gutierrez, P. M. (2004). An investigation of differences between selfinjurious behavior and suicide attempts in a sample of adolescents. Suicide and LifeThreatening Behavior. doi:10.1521/suli.34.1.12.27769.

Murphy, M. J., Mermelstein, L. C., Edwards, K. M., \& Gidycz, C. A. (2012). The benefits of dispositional mindfulness in physical health: A longitudinal study of female college students. Journal of American College Health. doi:10.1080/07448481.2011.629260.

Nicholls, A. R., Polman, R., \& Levy, A. R. (2010). Coping self-efficacy, pre-competitive anxiety, and subjective performance among athletes. European Journal of Sport Science. doi:10.1080/17461390903271592.

Nock, M. K., \& Mendes, W. B. (2008). Physiological arousal, distress tolerance, and social problem-solving deficits among adolescent self-injurers. Journal of Consulting and Clinical Psychology. doi:10.1037/0022-006X.76.1.28.

Park, T., Reilly-Spong, M., \& Gross, C. R. (2013). Mindfulness: A systematic review of instruments to measure an emergent patient-reported outcome (PRO). Quality of Life Research. doi:10.1007/s11136-013-0395-8.

Preacher, K. J., \& Hayes, A. F. (2004). SPSS and SAS procedures for estimating indirect effects in simple mediation models. Behavior Research Methods, Instruments, \& Computers. doi:10.3758/BF03206553.

Rasmussen, M. K., \& Pidgeon, A. M. (2011). The direct and indirect benefits of dispositional mindfulness on self-esteem and social anxiety. Anxiety, Stress and Coping, doi. doi:10.1080/10615806.2010.515681. 
This is a post-peer-review, pre-copyedit version of an article published in 'Mindfulness'. The final authenticated version is available online at: https://doi.org/10.1007/s12671-016-0555-3. The following terms of use apply: https://www.springer.com/gp/open

Shapiro, S. J., Brown, K. W., Thoresen, C., \& Plante, T. G. (2011). The moderation of mindfulness-based stress reduction effects by trait mindfulness: Results from a randomized controlled trial. Journal of Clinical Psychology. doi:10.1002/jclp.20761.

Siegling, A. B., \& Petrides, K. V. (2014). Measures of trait mindfulness: Convergent validity, shared dimensionality, and linkages to the five-factor model. Frontiers in Psychology. doi:10.3389/fpsyg.2014.01164.

Simeon, D., \& Favazza, A. R. (2001). Self- injurious behaviors: Phenomenology and assessment. In D. Simeon \& E. Hollander (Eds.), Self- injurious behaviors: Assessment and treatment (pp. 1-28). Washington, DC: American Psychiatric Press.

Stillman, C. M., Feldman, H., Wambach, C. G., Howard, J. H., Jr., \& Howard, D. V. (2014). Dispositional mindfulness is associated with reduced implicit learning. Consciousness and Cognition. doi:10.1016/j.concog.2014.07.002.

Tabachnick, B. G., \& Fidell, L. S. (2013). Using multivariate statistics (6th ed.). Boston: Pearson.

Tran, U. S., Cebolla, A., Gluck, T. M., Soler, J., Garcia-Campayo, J., \& von Moy, T. (2014). The serenity of the meditating mind: A cross-cultural psychometric study on a two-factor higher order structure of mindfulness, its effects, and mechanisms related to mental health among experience meditators. Public Library of Science. doi:10.1371/journal.pone.0110192. 
This is a post-peer-review, pre-copyedit version of an article published in 'Mindfulness'. The final authenticated version is available online at: https://doi.org/10.1007/s12671-016-0555-3. The following terms of use apply: https://www.springer.com/gp/open

van den Bosch, L. M. C., Koeter, M. W. J., Stinjen, T., Verheul, R., \& van den Brink, W. (2005). Sustained efficacy of dialectical behaviour therapy for borderline personality disorder. Behaviour Research and Therapy. doi:10.1016/j.brat.2004.09.008.

Vujanovic, A. A., Bonn-Miller, M. O., Bernstein, A., McKee, L. G., \& Zvolensky, M. J. (2010). Incremental validity of mindfulness skills in relation to emotional dysregulation among a young adult community sample. Cognitive Behaviour Therapy. doi:10.1080/16506070903441630.

Walsh, B. W. (2012). Treating self-injury: A practical guide. New York: Guildford Press.

Whitlock, J., Eckenrode, J., \& Silverman, D. (2006). Self-injurious behaviors in a college population. Pediatrics. doi:10.1542/peds.2005-2543.

Wupperman, P., Fickling, M., Klemanski, D. H., Berking, M., \& Whitman, J. B. (2013). Borderline personality features and harmful dysregulated behavior: The meditational effect of mindfulness. Journal of Clinical Psychology. doi:10.1002/jclp.21969.

Yusainy, C., \& Lawrence, C. (2014). Relating mindfulness and self-control to harm to the self and to others. Personality and Individual Differences. doi:10.1016/j.paid.2014.02.015. 
This is a post-peer-review, pre-copyedit version of an article published in 'Mindfulness'. The final authenticated version is available online at: https://doi.org/10.1007/s12671-016-0555-3. The following terms of use apply: https://www.springer.com/gp/open

\section{Tables and Figures}

Table 1 Summary of the means and standard deviations of cse and mindfulness as a function of group membership

\begin{tabular}{|l|l|l|l|}
\hline Total sample & & & \\
\hline Variable & $\mathrm{M}$ & SD & $n$ \\
\hline 1. CSE & 113.33 & 35.93 & 80 \\
\hline 2. Mindfulness & 53.04 & 12.35 & 81 \\
\hline NSSI group & & & \\
\hline Variable & $\mathrm{M}$ & SD & $n$ \\
\hline 1. CSE & 96.73 & 31.97 & 30 \\
\hline 2. Mindfulness & 48.68 & 10.71 & 31 \\
\hline Comparison group & & & \\
\hline Variable & $\mathrm{M}$ & SD & $n$ \\
\hline 1. CSE & 123.28 & 34.75 & 50 \\
\hline 2. Mindfulness & 55.74 & 12.62 & 50 \\
\hline CSE coping self-efficacy & & \\
\hline
\end{tabular}

\section{Figure 1}

a

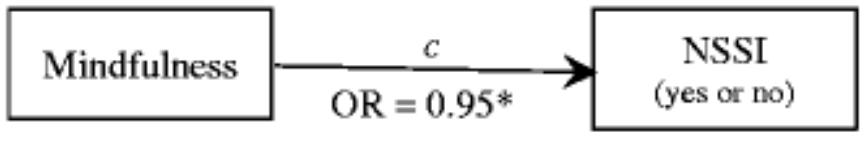

b

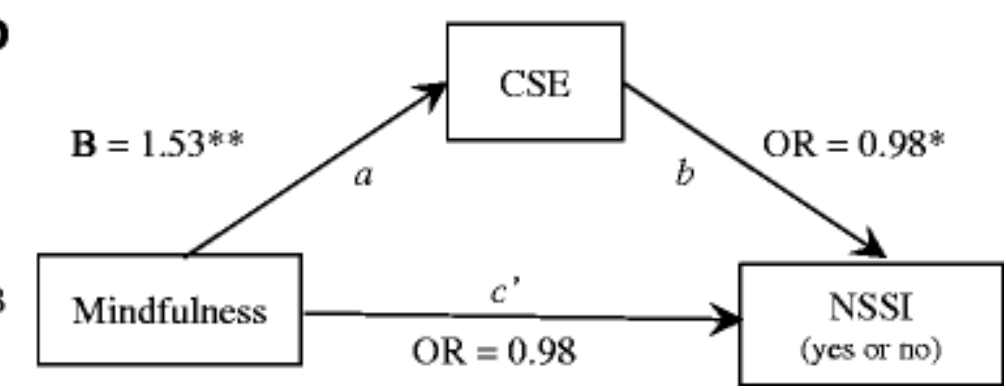

A three-variable mediation model. a The total effect model of mindfulness on NSSI status. $b$ The simple mediation model with coping self-efficacy as a mediator between mindfulness and NSSI status. B unstandardized regression coefficient; OR odds ratio; CSE coping selfefficacy; c total effect of mindfulness on NSSI status without controlling for coping self- 
This is a post-peer-review, pre-copyedit version of an article published in 'Mindfulness'. The final authenticated version is available online at: https://doi.org/10.1007/s12671-016-0555-3. The following terms of use apply: https://www.springer.com/gp/open

efficacy; c' direct effect of mindfulness on NSSI status after controlling for coping selfefficacy; $* * p<0.001 ; * \mathrm{p}<0.05$

Table 2 Unstandardized regression coefficients, standard errors, odds ratio, and confidence intervals of the simple mediation model

\begin{tabular}{|l|l|l|l|l|l|l|}
\hline & & & & & & \multicolumn{2}{|c|}{$\begin{array}{c}\text { Confidence } \\
\text { intervals }\end{array}$} \\
\hline Path/effect & B & SE & Wald & OR & Lower & Upper \\
\hline c (Mindfulness $\rightarrow$ NSSI) & -0.05 & 0.0 & 5.90 & $0.95^{*}$ & 0.91 & 0.99 \\
\hline a (Mindfulness $\rightarrow$ CSE) & $1.53^{* *}$ & 0.3 & -- & -- & 0.93 & 2.13 \\
\hline b (CSE $\rightarrow$ NSSI) & -0.02 & 0.0 & 4.67 & $0.98^{*}$ & 0.96 & 0.99 \\
\hline c' & -0.02 & 0.0 & 0.61 & 0.98 & 0.94 & 1.03 \\
\hline a x b & -0.03 & 0.0 & -- & -- & -0.0736 & -0.002 \\
& & & & & & \\
\hline
\end{tabular}

NSSI NSSI status, CSE coping self-efficacy, B unstandardized regression coefficient, OR odds ratio, $\mathrm{c}$ the total effect of mindfulness on NSSI status without controlling for coping selfefficacy; c' direct effect of mindfulness on NSSI status after controlling for coping selfefficacy, a x b indirect effect of coping self-efficacy in the relation between mindfulness and NSSI status

$* * \mathrm{p}<0.001 ; * \mathrm{p}<0.05$

A Confidence interval obtained with the bootstrap bias-corrected method 